\title{
Intracavernous internal carotid artery pseudoaneurysm
}

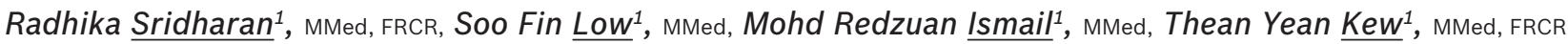

\begin{abstract}
Epistaxis is commonly encountered in otorhinolaryngologic practice. However, severe and recurrent epistaxis is rarely seen, especially that originating from a pseudoaneurysm of the intracavernous internal carotid artery (ICA). We herein present the case of a 32-year-old man who was involved in a motor vehicle accident and subsequently developed recurrent episodes of profuse epistaxis for the next three months, which required blood transfusion and nasal packing to control the bleeding. Computed tomography angiography revealed a large intracavernous ICA pseudoaneurysm measuring $1.7 \mathrm{~cm} \times 1.2 \mathrm{~cm} \times 1.0 \mathrm{~cm}$. The patient underwent emergent four-vessel angiography and coil embolisation and was discharged one week later without any episode of bleeding. He remained asymptomatic after three-month and one-year intervals. This case report highlights a large intracavernous ICA pseudoaneurysm as a rare cause of epistaxis, which requires a high index of suspicion in the right clinical setting and emergent endovascular treatment to prevent mortality.
\end{abstract}

Keywords: carotid, endovascular, epistaxis, intracavernous, pseudoaneurysm

\section{INTRODUCTION}

Epistaxis is the commonest emergency in otorhinolaryngologic practice and often causes great distress in both patients and clinicians. More than half of the general population would have experienced an episode of epistaxis of varying severity. ${ }^{(1)}$ Medical intervention is only needed in approximately $6 \%$ of these patients, as cases of massive epistaxis are rare.(1) Digital trauma to the nasal mucosa, extreme dryness of the nasal cavity, hypertension, anticoagulation and blood dyscrasia are some common causes of epistaxis. ${ }^{(2)}$ Trauma and vascular abnormalities account for less than $5 \%$ of all cases of epistaxis. ${ }^{(3)}$ Although head trauma is a well-known cause of epistaxis, epistaxis resulting from a traumatic pseudoaneurysm of the internal carotid artery (ICA) is relatively rare. This condition can result in mortality if unrecognised. Management of this condition is unique from that of epistaxes of other causes. Herein, we describe a post-traumatic ICA pseudoaneurysm, in which endovascular treatment with preservation of the parent artery was successful.

\section{CASE REPORT}

A 32-year-old man was referred to our institution for further management of a cavernous ICA pseudoaneurysm. The patient was previously involved in a road traffic accident and had sustained head, facial and abdominal injuries one month prior to presentation. He had undergone laparotomy for his abdominal injuries and was discharged after a month-long hospital stay, with dental and orthopaedic follow-ups. Unfortunately, two months following his discharge from hospital, he presented to the emergency department with two episodes of epistaxis. However, the bleeding was minimal and stopped spontaneously without requiring in-patient hospitalisation. In the third month after the patient's discharge, he again presented with two episodes of torrential epistaxis.

The patient's haemoglobin level on admission was $4.7 \mathrm{~g} / \mathrm{dL}$. His prothrombin time (PT) and activated partial thromboplastin

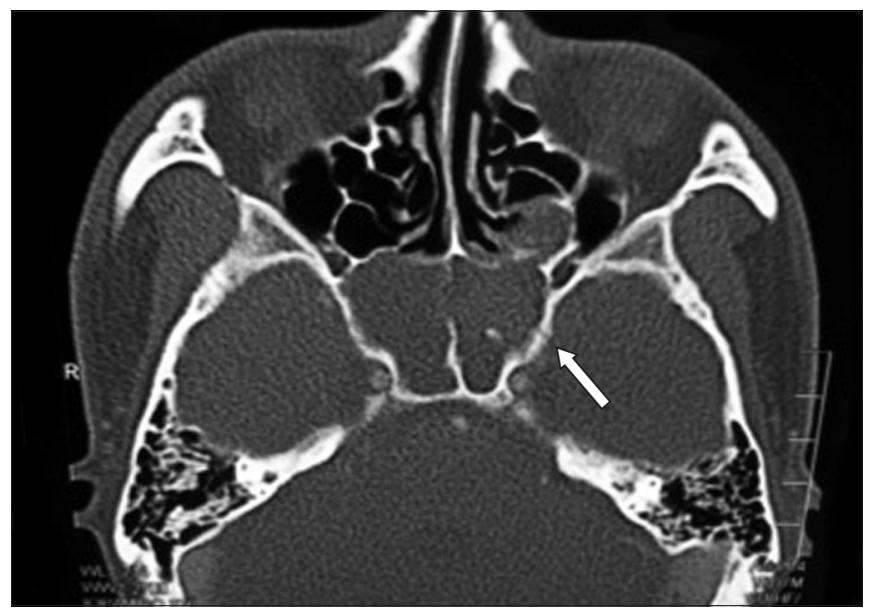

Fig. 1 CT angiogram shows a linear fracture of the lateral wall of the left sphenoid sinus (arrow).

time (aPTT) were prolonged, with an international normalised ratio (INR) of 5.93. He received blood transfusion, and his coagulation profile was subsequently corrected. A $30^{\circ}$ rigid endoscopy was performed, revealing a swelling with bluish discolouration originating from the left side of the nasopharynx. Blood was also seen trailing down from a vascular lesion in the region. Clinical impression of the lesion was promptly made, and nasal packing was applied to help control the bleeding. Subsequently, computed tomography (CT) angiography was done to help confirm the suspicion of a vascular lesion in the nasopharynx and to exclude other differentials (e.g. vascular tumour). CT angiography showed a linear fracture involving the lateral wall of the left sphenoid sinus (Fig. 1) with a prominent cavernous ICA pseudoaneurysm projecting into the affected sphenoid sinus (Fig. 2).

The patient was referred to our institution for therapeutic embolisation. An emergent four-vessel cerebral angiography was done, revealing a large pseudoaneurysm measuring $1.7 \mathrm{~cm} \times$ $1.2 \mathrm{~cm} \times 1.0 \mathrm{~cm}$ and pointed anteroinferiorly in the cavernous

${ }_{1}^{1}$ Department of Radiology, Universiti Kebangsaan Malaysia Medical Centre, Kuala Lumpur, Malaysia

Correspondence: Dr Radhika Sridharan, Consultant Radiologist and Senior Lecturer, Department of Radiology, Universiti Kebangsaan Malaysia Medical Centre, Jalan Yaakob Latiff, 56000 Cheras, Kuala Lumpur, Malaysia. sridharan.radhika@gmail.com 


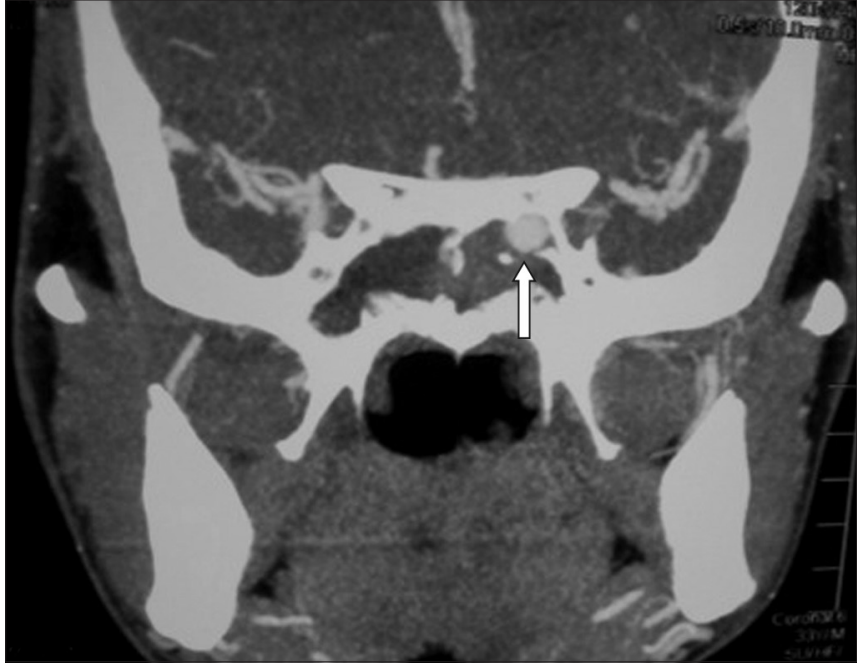

Fig. 2 CT angiogram shows a well-demarcated, avidly enhancing lesion in the left sphenoid sinus (arrow), suggestive of a pseudoaneurysm of the intracavernous portion of the left ICA

portion of the left ICA (Fig. 3). The rest of the vessels were normal. An occlusion test of the left cervical ICA was performed to assess the cross-flow from the contralateral ICA, in case the carotid artery needed to be sacrificed.

Despite a successful occlusion test, preserving the patency of the ICA was the desired goal. Coil embolisation was done using an Excelsior SL-10 microcatheter (Boston Scientific, Natick, Massachusetts, USA) and Transcend 14 microguidewire (Boston Scientific, Natick, Massachusetts, USA). No problems were encountered during the navigation. Multiple coils were deployed, including two- and three-dimensional intracranial Target ${ }^{\circledR}$ Detachable Coils (Boston Scientific/Stryker Neurovascular, Fremont, California, USA). Post-procedural angiography showed successful occlusion of the aneurysmal neck (Fig. 4). The patient had no history of recurrent epistaxis; he had his nasal packs removed and was discharged uneventfully after one week. He was seen in the clinic after three-month and one-year intervals and remained symptom-free.

\section{DISCUSSION}

Only $3 \%-5 \%$ of all intracranial aneurysms originate from cavernous ICA. ${ }^{(4)}$ Most of these cases are attributed to trauma, with an associated mortality rate of up to $50 \% .{ }^{(5)}$ Trauma-induced vessel wall defect in the presence of constant arterial pressure would result in blood dissecting into the surroundings and forming a perfused sac (i.e. pseudoaneurysm). A direct communication exists between the lumen of the vessel and the sac of the pseudoaneurysm, with blood flowing through the defect in the vessel wall. The perfused aneurysmal sac is contained by the adventitia or surrounding soft tissues. The risk of rupture is therefore higher in a pseudoaneurysm than in an aneurysm of a similar size, due to inadequate support of the pseudoaneurysm wall.

Although most cases occur after severe head injuries associated with skull base fractures, postoperative and spontaneous nontraumatic pseudoaneurysms have been described. These pseudoaneurysms may either be asymptomatic or tear into the sphenoid sinus and present with delayed torrential epistaxis. ${ }^{(6)}$ Recurrent epistaxis occurs, as a small, full thickness tear of the ICA can temporarily seal off from a haematoma within the enclosure of the sphenoid sinus. ${ }^{(7)}$ The cavernous ICA has been observed to protrude into the lateral wall of the sphenoid sinus in $70 \%$ of cadaveric specimens, as it is intimately related to this sinus. ${ }^{(8)}$ The bony covering over the cavernous ICA within the sphenoid sinus measures less than $1 \mathrm{~mm}$ in $66 \%$ of cases, and does not exist in $4 \%$ of them. ${ }^{(8)}$ The thin tissue interface between these structures therefore predisposes the sphenoid sinus for ICA injury in cases of trauma and sphenoid sinus surgery. ${ }^{(2)}$ The intracavernous ICA is also relatively fixed in its position, making it likely to tear during an acceleration-deceleration injury, resulting in either a fistulous communication into the cavernous sinus or the formation of a pseudoaneurysm in the sphenoid sinus. The anteromedially directed aneurysmal sac in the present case, situated away from the laterally situated cranial nerves adjacent to the cavernous sinus, probably accounted for the absence of concurrent cranial nerve palsies in our patient.

This condition (i.e. intracavernous ICA pseudoaneurysm) typically results in delayed, repetitive and massive epistaxis occurring within three weeks of the initial insult. Refractory haemorrhage could even be delayed by months or years. ${ }^{(9)}$ The initial episodes of epistaxis are typically not severe, but they tend to recur with increasing severity that can lead to rapid exsanguination. Diagnostic and therapeutic delays may occur, as the initial bleeding episode may not be particularly worrisome. ${ }^{(9)}$ The pathogenesis of delayed epistaxis could be attributed to the time taken for the arterial pulsatile pressure to weaken the injured vessel wall and erode through the sphenoid sinus. As pseudoaneurysms lack a true wall, the size of the sac would also usually increase with time.

Haematoma in the sphenoid sinus may suggest a dehiscent sinus wall, and any fractures of the sphenoid sinus, as seen in our patient, or sella turcica should alert surgeons and radiologists to a possible traumatic intracavernous ICA pseudoaneurysm. Therefore, a high index of suspicion for diagnosis of this condition is warranted. Carotid angiography is the gold standard for the elicitation of the diagnosis, and is mandatory in order to determine the feasibility of endovascular therapy. ${ }^{(10)}$ Special attention to the anatomy of the aneurysm, with thorough analysis of its geometrical dimensions, is required to decide whether the aneurysm can be obliterated in isolation while preserving the parent artery. Nevertheless, three-dimensional CT angiography is a less invasive alternative than conventional catheter angiography and is usually done as the initial investigation. CT angiography is rapid and has the ability to detect a pseudoaneurysm, assess the surrounding anatomical bony structures, as well as aid in planning further management (e.g. open surgery or endovascular embolisation). Magnetic resonance imaging can also provide the initial information needed to suggest the possibility of an ICA pseudoaneurysm. ${ }^{(10)}$

Early treatment of ICA pseudoaneurysm cannot be overemphasised. The mortality rate of post-traumatic aneurysms with rupture into the sphenoid sinus ranges from $30 \%$ to $50 \%{ }^{(5)}$ Emergent control of epistaxis will require nasal packing, allowing time for definitive neuroradiologic investigations and treatment. Endovascular techniques have taken precedence over surgical 

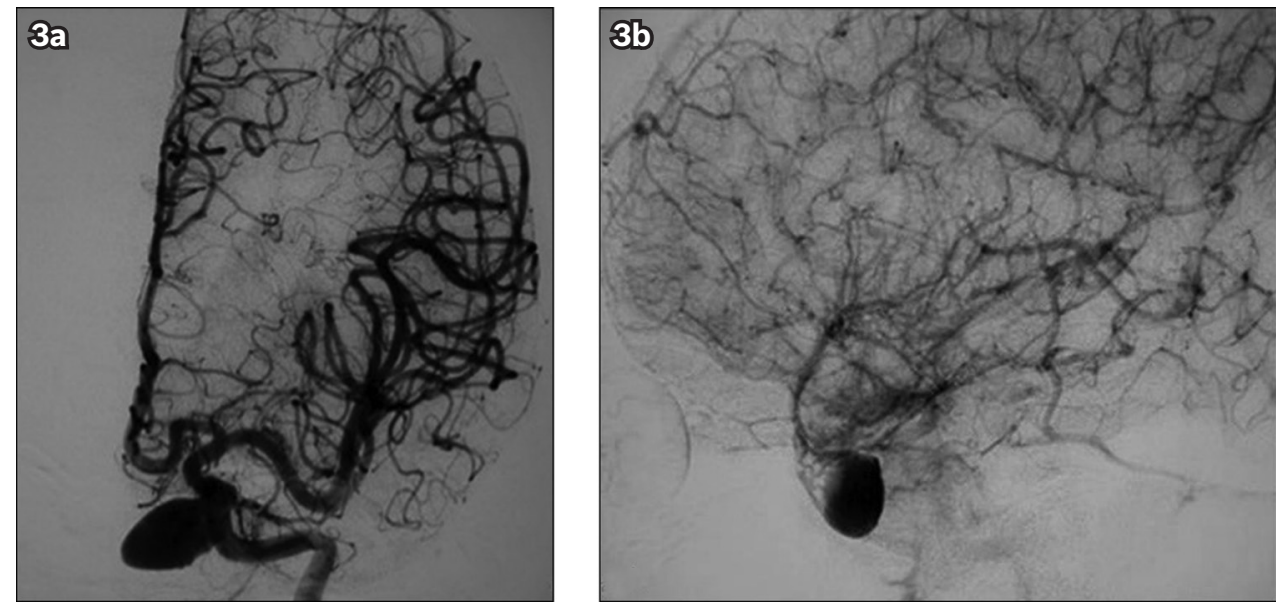

Fig. 3 Catheter angiograms show the (a) anterior-posterior and (b) lateral views of the left ICA, confirming the presence of the pseudoaneurysm.

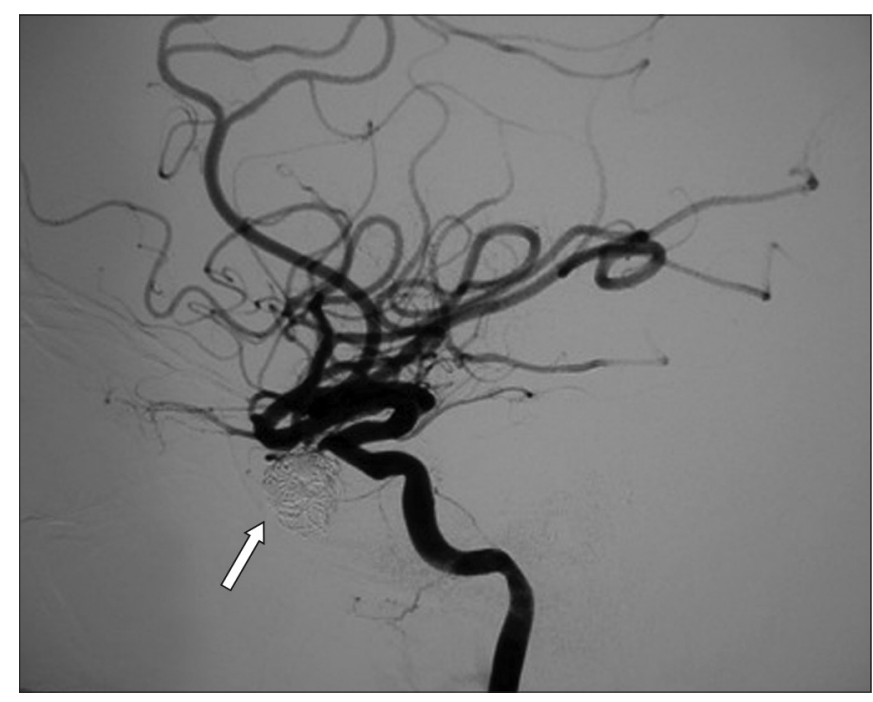

Fig. 4 Post-coil embolisation angiogram of the left ICA shows successful occlusion of the pseudoaneurysm (arrow).

management of cavernous ICA pseudoaneurysm, as the location of the vessel at the skull base, surrounded by the adjacent sphenoid bone, cavernous sinus and cranial nerves, complicates direct surgical approach.

Several technical approaches have been advocated for the management of traumatic ICA pseudoaneurysms, including sacrificing the parent artery either surgically (e.g. clipping, wrapping, trapping and carotid artery ligation) or endovascularly. However, sacrificing the parent artery may not be tolerated by the patient and could result in cerebral hypoperfusion and ischaemia.

In spite of angiography showing good crossflow from the contralateral cerebral hemisphere during the balloon occlusion test, parent artery occlusion may still put the patient at risk of stroke. The stroke rate among patients who undergo an abrupt ligation has been reported to be $26 \%$, whereas the stroke rate among patients who pass the balloon occlusion test is only $3 \% .{ }^{(11)}$ In another study, $5 \%-20 \%$ of patients who pass the balloon occlusion test developed infarction after permanent occlusion of the affected carotid artery. ${ }^{(12)}$ Endovascular occlusion of the ICA using coils may also impose a small risk of thromboembolic stroke due to the slow flow of blood across the incompletely occluded artery. ${ }^{(13)}$
Nevertheless, endovascular occlusion of the pseudoaneurysm, while preserving the parent artery, is currently the favoured method. Generally, a saccular aneurysm with a small neck can be embolised with coils, as in our patient. Although detachable coils and balloons run a risk of dislodgement through the fragile wall of the pseudoaneurysm, the bony confines of the sphenoid sinus may provide further support to the nest of coils placed in the pseudoaneurysm. ${ }^{(13)}$ The latent period between the initial injury and treatment in our patient has permitted maturing of the walls of the pseudoaneurysm with fibroblastic elements, thereby providing support for the coil mesh. ${ }^{(13)}$ Our patient remained asymptomatic one year post embolisation. Attempts to coil the pseudoaneurysm during the acute phase may be associated with a higher level of risk of rupture. Urgent stent placement is a viable treatment while preserving the parent artery. However, in some complex pseudoaneurysms and difficult ICA anatomy, parent vessel occlusion or trapping are acceptable alternatives.

Endosaccular coiling or detachable balloon placement has been reported to be reasonably safe and successful in occluding the pseudoaneurysm while preserving the parent artery. ${ }^{(13,14)}$ We believe that coil embolisation of the aneurysmal sac has an advantage over balloon embolisation in our case. Coils can absorb the haemodynamic forces caused by pulsatile blood flow better than the more rigid balloon that may transmit these stresses to the wall of the pseudoaneurysm. ${ }^{(13)}$ More recently, graft stents have been used to successfully treat ICA pseudoaneurysms. ${ }^{(15)}$ Current endovascular technology includes the use of stents that preserve the parent artery by either stent deployment or the concurrent use of stents and detachable coils for the treatment of wide-necked pseudoaneurysms, which are not amenable to conventional unsupported coil embolisation. However, there are disadvantages to the introduction of an endovascular stent, particularly in our patient, as its usage potentiates the risk of additional injury to the vessel wall and may result in stent thrombosis. The stents are relatively inflexible after deployment and may give rise to possible complications, such as dissection or delayed intimal hyperplasia, especially when deployed in tortuous arterial segments.

In summary, although intracavernous pseudoaneurysms are extremely rare among isolated sphenoid fractures, it 
must be considered in the differential diagnosis when the patient has refractory epistaxis with a previous history of head trauma. As these lesions are potentially lethal, early detection, together with emergent endovascular therapeutic procedures, is imperative. Our present case demonstrates that ICA pseudoaneurysm can be safely and effectively treated via coiling, provided that due consideration is given to the anatomic location of the pseudoaneurysm and the timing of the treatment.

\section{ACKNOWLEDGEMENTS}

We extend special gratitude to the Dean and Director, Faculty of Medicine, Universiti Kebangsaan Malaysia Medical Centre, Malaysia, for the approval to conduct the above case study. We also thank Nooradila Zolkiflee and Siti Farizwana Mohd Ridzwan for their assistance in the preparation of the manuscript.

\section{REFERENCES}

1. Elahi MM, Parnes LS, Fox AJ, Pelz DM, Lee DH. Therapeutic embolization in the treatment of intractable epistaxis. Arch Otolaryngol Head Neck Surg 1995; 121:65-9.

2. Chen D, Concus AP, Halbach VV, Cheung SW. Epistaxis originating from traumatic pseudoaneurysm of the internal carotid artery: diagnosis and endovascular therapy. Laryngoscope 1998; 108:326-31.

3. Juselius $\mathrm{H}$. Epistaxis. A clinical study of 1,724 patients. J Laryngol Otol $1974 ; 88: 317-27$.
4. Bars HW, Blackwood W, Meadows SP. Intracavernous carotid aneurysms. A clinical-pathological report. Brain 1971; 94:607-22.

5. Wang AN, Winfield JA, Güçer G. Traumatic internal carotid aneurysm with rupture into the sphenoid sinus. Surg Neurol 1986; 25:77-81.

6. Bhatoe HS, Suryanarayana KV, Gill HS. Recurrent massive epistaxis due to traumatic intracavernous internal carotid artery aneurysm. J Laryngol Otol 1995; 109:650-2.

7. Chandy MJ, Rajshekhar V. Nontraumatic intracavernous carotid aneurysm presenting with epistaxis. J Laryngol Otol 1989; 103:425-6.

8. Renn WH, Rhoton AL Jr. Microsurgical anatomy of the sellar region. J Neurosurg 1975; 43:288-98.

9. Chambers EF, Rosenblum AE, Norman D, Newton TH. Traumatic aneurysms of cavernous internal carotid artery with secondary epistaxis. Am J Neuroradiol 1981; 2:405-9.

10. Kim JY, Farkas J, Putman CM, Varvares M. Paraclinoid internal carotid artery aneurysm presenting as massive epistaxis. Ann Otol Rhinol Laryngol 2000; 109:782-6.

11. Mathis JM, Barr JD, Jungreis CA, et al. Temporary balloon test occlusion of the internal carotid artery: experience in 500 cases. Am J Neuroradiol 1995; 16:749-54.

12. de Vries EJ, Sekhar LN, Horton JA, et al. A new method to predict safe resection of the internal carotid artery. Laryngoscope 1990; 100:85-8.

13. Lempert TE, Halbach VV, Higashida RT, et al. Endovascular treatment of pseudoaneurysms with electrolytically detachable coils. AJNR Am J Neuroradiol 1998; 19:907-11.

14. Tantana S, Pilla TJ, Awwad EE, Smith KR. Balloon embolization of a traumatic carotid-ophthalmic pseudoaneurysm with control of the epistaxis and preservation of the internal carotid artery. AJNR Am J Neuroradiol 1987; 8:923-4.

15. Maras D, Lioupis C, Magoufis G, et al. Covered stent-graft treatment of traumatic internal carotid pseudoaneurysms: a review. Cardiovasc Intervent Radiol 2006; 29:958-68. 